Association for Information Systems AIS Electronic Library (AISeL)

Wirtschaftsinformatik Proceedings 2003

Wirtschaftsinformatik

September 2003

\title{
Portal-Engineering - Anforderungen an die Entwicklung komplexer Unternehmensportale
}

Michael Amberg

Universität Erlangen-Nürnberg

Ulrich Remus

Universität Erlangen-Nürnberg, ulrich.remus@wiso.uni-erlangen.de

Jochen Holzner

aveniox business consulting $\mathrm{GmbH}$

Follow this and additional works at: http://aisel.aisnet.org/wi2003

\section{Recommended Citation}

Amberg, Michael; Remus, Ulrich; and Holzner, Jochen, "Portal-Engineering - Anforderungen an die Entwicklung komplexer Unternehmensportale" (2003). Wirtschaftsinformatik Proceedings 2003. 93.

http://aisel.aisnet.org/wi2003/93

This material is brought to you by the Wirtschaftsinformatik at AIS Electronic Library (AISeL). It has been accepted for inclusion in Wirtschaftsinformatik Proceedings 2003 by an authorized administrator of AIS Electronic Library (AISeL). For more information, please contact elibrary@aisnet.org. 
In: Uhr, Wolfgang, Esswein, Werner \& Schoop, Eric (Hg.) 2003. Wirtschaftsinformatik 2003: Medien - Märkte - Mobilität, 2 Bde. Heidelberg: Physica-Verlag

ISBN: 3-7908-0111-9 (Band 1)

ISBN: 3-7908-0116-X (Band 2)

(C) Physica-Verlag Heidelberg 2003 


\title{
Portal-Engineering - Anforderungen an die Entwicklung komplexer Unternehmensportale
}

\author{
Michael Amberg, Ulrich Remus \\ Universität Erlangen-Nürnberg \\ Jochen Holzner \\ aveniox business consulting $\mathrm{GmbH}$
}

Zusammenfassung: Unternehmensportale für das betriebliche Informations- und Wissensmanagement sind hochkomplex: Mehrere hundert Benutzergruppen mit unterschiedlichsten Informationsbedürfnissen greifen auf zahlreiche Informationsquellen zu. Die Zahl der Anwendungsbausteine innerhalb von Portalen (Portlets), welche interne und externe Informationen sowie betriebliches Wissen bereitstellen, kann in die Tausende gehen.

Ein Portal-Engineering verfolgt das Ziel, Unternehmens- und insbesondere Mitarbeiterportale (Business-to-Employee-Portals) methodisch unterstützt zu planen, um sie effizienter und effektiver als bisher implementieren zu können. In diesem Beitrag werden die wesentlichen Rahmenbedingungen und Anforderungen diskutiert, die an ein Portal-Engineering gestellt werden. Anschließend wird aufgezeigt, inwieweit bestehende Ansätze diese Anforderungen erfüllen. Darauf aufbauend können mögliche Komponenten und Inhalte eines Instrumentariums zur ingenieurmäßigen Konzeption und Einführung von Unternehmensportalen entwickelt werden.

Schlüsselworte: Unternehmensportal, Mitarbeiterportal, Business-to-EmployeePortal, Referenzmodelle, Architekturmodelle, Portal-Engineering, Portal Management, Informations- und Wissensmanagement

\section{Unternehmensportale als Integrationsplattformen der Zukunft}

\section{1 Überblick und Motivation}

Mitarbeiter- und Unternehmensportale integrieren Anwendungen, Dienste und Inhalte aus vielen verschiedenen Informationsquellen auf einer Oberfläche am Arbeitsplatz eines Mitarbeiters, Kunden oder Lieferanten (Single Point of Access). 
Die webbasierte Portaltechnologie bietet völlig neue organisatorische und informationstechnische Möglichkeiten, wie z. B. die Personalisierung von Informationen, eine schnelle und hochaktuelle Verteilung von Wissen sowie die flexible $\mathrm{Zu}$ weisung von Benutzerrollen. Daher entwickeln sich Unternehmensportale zu einer wichtigen Integrationsplattform für die Optimierung der betrieblichen Informations- und Wissenslogistik in Unternehmen jeglicher Größe. Der gehobene Mittelstand, Großunternehmen und insbesondere Konzerne beginnen gegenwärtig stark in Unternehmensportale, insbesondere in Mitarbeiterportale (Business-toEmployee-Portals), zu investieren. Siemens beispielsweise setzt die Portaltechnologie von SAP künftig konzernweit für alle rund 400.000 Mitarbeiter ein. Ebenso investieren Firmen der Automobilindustrie wie DaimlerChrysler oder Unternehmen der Luftfahrt, wie z. B. die Lufthansa, hohe Summen in Mitarbeiter-, Kunden- und Lieferantenportale.

Unternehmen versprechen sich mit der Einführung von Portalen einige Potenziale. Um sich zu einer zentralen Informations- und Anwendungsplattform für die betriebliche Informations- und Wissenslogistik in Unternehmen verschiedener Größen, Branchen und Betriebstypen zu entwickeln, müssen Portale beliebig skalierbar und anpassbar an verschiedene Anwendungsszenarien sein. Sie müssen die Prozessintegration und -harmonisierung unterstützen und einen einheitlichen $\mathrm{Zu}$ gang zu Informationen bieten; außerdem müssen sich Anwendungen unterschiedlichster Softwarehersteller integrieren lassen. Eine weitere wichtige Eigenschaft ist die Personalisierung, die es erlaubt, das Portal für bestimmte Zielgruppen, Rollen und Aufgaben "maßzuschneidern" und an persönliche Profile anzupassen.

Erfahrungen aus Praxisprojekten zeigen, dass sich Portale zum heutigen Zeitpunkt nur äußerst aufwändig konzipieren, implementieren und administrieren lassen. Portalprojekte sind gegenwärtig kostenträchtig und ineffizient; die daraus resultierenden Anwendungssysteme sind schwer zu warten und nur unter größerem Aufwand erweiterbar. Dies mag einerseits an der großen Komplexität der Portale liegen: Mehrere hundert Benutzergruppen mit unterschiedlichsten Informationsbedürfnissen greifen auf Hunderte von Informationsquellen zu. Einige tausend Anwendungsbausteine innerhalb der Portale (Portlets) liefern interne und externe Informationen sowie betriebliches Wissen.

Andererseits müssen sich Portale schnell an geänderte Unternehmensanforderungen anpassen lassen. Gerade das Management komplexer Unternehmensportale ist unter dem Gesichtspunkt sorgfältiger, langfristig gültiger Planung und der geforderten Flexibilität und Adaptivität dieser neuartigen Anwendungssysteme noch überaus schwierig.

Unternehmen müssen bei der Planung, Implementierung und Konfiguration von Unternehmensportalen immer wieder von Neuem beginnen, da es an etablierten Instrumentarien zur systematischen, modellbasierten Konzeption und Einführung von Portalen fehlt. Aufeinander abgestimmte Vorgehensweisen, Instrumente, Me- 
thoden und branchenspezifische Referenz-Anwendungsarchitekturen sind momentan noch nicht vorhanden.

Dieser Beitrag verfolgt das Ziel, die wesentlichen Problemfelder bei der systematischen, ingenieurmäßigen Entwicklung von kommerziellen Unternehmens- und Mitarbeiterportalen, sowie darauf aufbauend Anforderungen an Portalentwicklungsansätze herauszuarbeiten. Im Vordergrund stehen Anforderungen an integrierte Modellierungs- und Entwicklungsinstrumente, welche die Konzeption, Einführung und Anpassung von kommerziellen Standardportalprodukten, wie z. B. Bea WebLogic, IBM Portal Server, Plumtree Corporate Portal, Hyperwave Information Portal oder SAP Enterprise Portal, durchgängig unterstützen. Diese leiten sich sowohl aus der Recherche in einschlägiger Literatur als auch aus den eigenen Erfahrungen in Portalprojekten ab. Dazu werden zunächst in Kap. 1.2 Klassifikationen und mögliche Einsatzfelder von Unternehmensportalen aufgezeigt. Kap. 6 identifiziert die Komplexität von Portalen und die mehrstufige PortalWertschöpfungskette als die zentralen Problembereiche. Davon ausgehend werden in Kap. 3 Anforderungen an Vorgehensweisen, Methoden und Werkzeuge zur Entwicklung von Portalen abgeleitet und bereits Hinweise zur Lösung gegeben. Kap. 4 beleuchtet einige ausgewählte Ansätze zum Portal-Engineering hinsichtlich der vorher aufgestellten Anforderungen. Kap. 5 fasst die wichtigsten Ergebnisse zusammen und zeigt mögliche Forschungs- und Entwicklungsziele auf.

\subsection{Einsatzfelder und Klassifikation von Unternehmensportalen}

Ähnlich wie die betriebswirtschaftlichen Standardsysteme Anfang der 70er Jahre stehen Unternehmensportale als Anwendungsplattformen für das betriebliche Informations- und Wissensmanagement heute erst am Anfang ihrer Marktentwicklung. Sie werden voraussichtlich in den nächsten Jahren eine vergleichbare Bedeutung für die Volkswirtschaft und für jedes einzelne Unternehmen erlangen wie die ERP-Software. Insbesondere große Unternehmen tätigen inzwischen bedeutende Investitionen in Unternehmensportale zur Vernetzung von Mitarbeitern, Kunden und Lieferanten.

Der gehobene Mittelstand sowie kleine und mittlere Unternehmen (KMU) werden in den nächsten Jahren vermehrt in Unternehmens- und Wissensportale investieren. Man beobachtet, dass sich ein eigener Markt für Portale entwickelt [Piro02]: Laut Meta Group werden diese Anwendungssysteme in 2003 auf Platz 1 bei Softwareprojekten rücken. Bis Ende 2003 wollen ca. 85\% der Global-2000Unternehmen ein Unternehmensportal einführen. Es gibt inzwischen viele Anbieter von Standardplattformen für den Aufbau solcher Portale [PFiF01], so z. B. die großen Portalanbieter SAP, IBM und Plumtree; aber auch zahlreiche kleinere Portalhersteller (ca. 50) agieren am Markt. Es existiert eine Vielzahl unterschiedlicher Portalsoftwarepakete, die durch Verknüpfung mit individuell entwickelten oder zugekauften Portlets, d. h. mit Softwarebausteinen und "Webservices", zu unter- 
nehmens- und branchenspezifischen Portalen ausgebaut werden können. So entstehen beispielsweise Knowledge Portals, Enterprise Information Portals, Corporate Portals, E-Business-Portals und geschäftsprozessbezogene Portale.

Aufgrund des breiten Anwendungsspektrums und der Entwicklungsgeschichte der Portalhersteller (Web Content Management, ERP, Wissensmanagement) verwundert es nicht, dass es bisher noch keine allgemeingültige Klassifikation für Portale gibt. Eine der möglichen Klassifikationen unterscheidet verschiedene aufeinander aufbauende Stufen und berücksichtigt so den unterschiedlichen Funktionsumfang (vgl. z. B. [ShTy98], [Delp99], [Fink99], [Fire99], [ScWi02], [Baue01]); man grenzt dann etwa Enterprise Information Portals (EIP), Enterprise Collaborative Portals (ECP), Enterprise Expertise Portals (EEP) sowie Enterprise Knowledge Portals (EKP) voneinander ab.

Eine andere Klassifizierung baut auf verschiedenen Kategorien auf und erweitert den Kontext auch auf unternehmensexterne Bereiche [Hind02]. So lassen sich Portale unterscheiden nach der Geschäftsbeziehung (B2B, B2E, B2C), nach Zielgruppen (Business, Enterprise, Consumer), den Anwendungsbereichen (Horizontal, Vertikal), dem Fokus (Information, Integration, Collaboration) und dem jeweiligen Zugang (Öffentlich, Privat). Danach können z. B. intern fokussierte Enterprise Portale, extern fokussierte Extended Relationship Management Portale oder auch Prozessportale zur Prozessintegration abgegrenzt werden.

Die Fülle an möglichen Portalkategorien begründet auch die Vielzahl an Modulen bzw. Portlets, auf die ein Portalbenutzer Zugriff haben kann. Die Ziele, die man mit dem Einsatz von Portalen verfolgt, sind demnach so verschieden wie ihre Ausrichtung. Wissenschaftliche Arbeiten im Portalbereich legen ihre Schwerpunkte meist auf die Portaltechnologie, auf die Portalarchitektur und auf die Entwicklung von Portalen [Bach00], [Jans01], [Gurz02]. Die Modellierung und Beherrschung der komplexen Systeme wurde bisher eher vernachlässigt [BaSe00].

\section{Problemfelder und Rahmenbedingungen bei der Entwicklung von Portalen}

\subsection{Komplexität von Portalen}

Das Hauptproblem bei der Konzeption und Implementierung von Unternehmensportalen ist ihre enorme Komplexität: diese ist nahezu vergleichbar mit der aufwändigen und langwierigen Parametrisierung von ERP-Systemen. Zentrale Einflussfaktoren auf die Komplexität sind erstens die vielfältigen potenziellen Anwendungsbereiche und damit die Breite der unterschiedlichen Einsatzfelder von Portalen und Teilportalen, zweitens die Vielzahl zu integrierender Portlets und 
drittens die vielfältigen Teilsichten, die bei der Implementierung von Portalen zu berücksichtigen sind.

a) Breite der Einsatzfelder: Unternehmensportale können für zahlreiche verschiedene Funktionsbereiche, Geschäftsprozesse und Querschnittsbereiche konzipiert werden und sollten zudem in einer annehmbaren Zeit implementiert sein. Projektteams haben es daher mit einer Vielfalt möglicher Unternehmensportale zu tun (vgl. Abbildung 1).

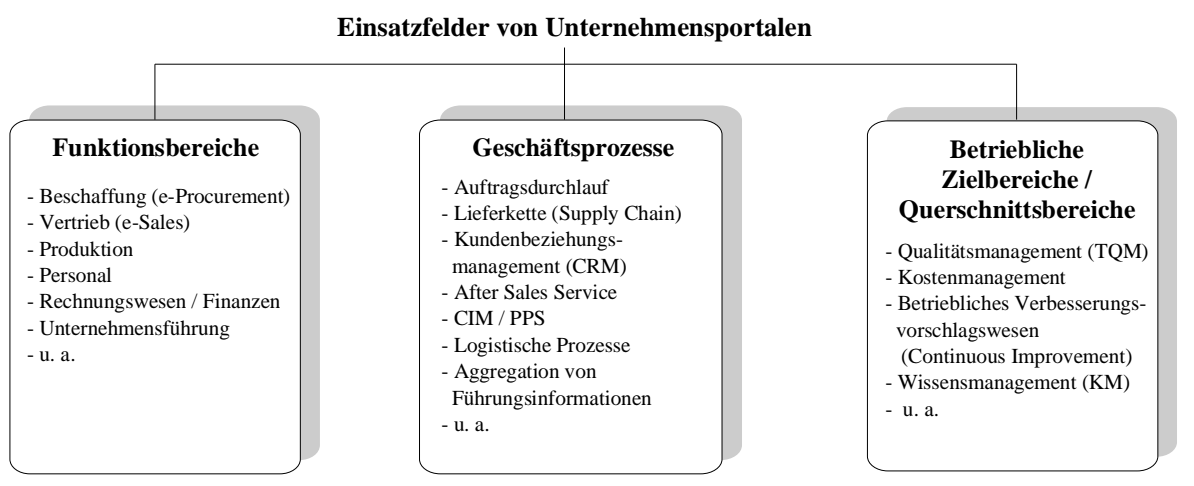

Abbildung 1: Hohe Bandbreite der Einsatzmöglichkeiten von Unternehmensportalen

b) Vielzahl von Portlets: Folgende Modellrechnung auf der Grundlage eines realen Mitarbeiterportals, welches bei einem Automobilhersteller eingesetzt wird, verdeutlicht die große Anzahl von Portlets, d. h. von einzelnen Portal-Bausteinen, die in einer Grundausbaustufe benötigt werden:

- Ein typischer Mitarbeiter dieses Automobilkonzerns greift täglich auf rund 50 Portlets zu, die teilweise vorgegeben sind (default-Portlets), teilweise aber von ihm am Bildschirm individuell ausgewählt und konfiguriert werden können.

- Das Unternehmen hat 7 Sparten mit durchschnittlich je 15 Abteilungen, in denen (annahmegemäß) rund 10 Benutzergruppen auf das Business-to Employee-Portal zugreifen können.

- Jede Benutzergruppe kann das Portal auf der Basis einiger tausend verfügbarer Portlets für seine Informations- und Wissensbedürfnisse einrichten.

Insgesamt gibt es somit konzernweit schätzungsweise 7 × 15 x $10=1050$ Benutzergruppen, die mit jeweils rund 50 - zum Teil verhältnismäßig funktionsreichen - Portlets arbeiten. Somit ist eine Fülle an Portlets bereitzustellen, die teils miteinander verknüpft sind, teils aus verschiedensten Informationsquellen gespeist und permanent aktualisiert werden müssen. Ein Großteil der Portlets ist zudem noch parametrisierbar und muss in mehreren Sprachen zur Verfügung stehen. 
c) Multiple Teilsichten: Eine weitere, dritte Schwierigkeit bei Portalprojekten neben der komplexen Struktur und der Vielzahl möglicher Portalkomponenten begründet sich durch die verschiedenen Teilsichten, die bei der Konzeption und Implementierung von Portalen zu bedenken sind:

- Es gibt vielfältige betriebliche und außerbetriebliche Benutzergruppen (Fachund Führungskräfte, Kunden, Lieferanten, Öffentlichkeit etc.).

- Jede Benutzergruppe hat jeweils spezifische Anforderungen an die Informationsstrukturen und -prozesse, die im Portal abgebildet werden sollen.

- Die internen und externen Informationsquellen können sich auf mehrere Hundert belaufen und sind über zahlreiche Schnittstellen miteinander verknüpft.

- Tausende von Anwendungsbausteinen (Portlets, Webservices) findet man relativ unsystematisiert und ,verstreut“ in der gesamten Softwarebranche, d. h. bei kleinen und mittelständischen Softwareanbietern, bei großen Softwaredienstleistern und bei Portalherstellern. Es ist nicht leicht zu entscheiden, ob Portlets im Anwenderbetrieb selbst realisiert oder besser zugekauft werden sollen, weil entsprechende zentrale, übergreifende Datenbanken mit einheitlichen Deskriptoren bzw. Suchmechanismen für Anwendungsbausteine fehlen.

Diese Komplexitätsproblematik bei Portalprojekten besteht unabhängig von der letztlich verwendeten Portalsoftware und -technologie.

\subsection{Mehrstufige Portal-Wertschöpfungskette}

Die zunehmende Komponentenorientierung hat einige Auswirkungen auf die Software-Industrie. Zukünftig werden die Software-Hersteller ihre große Fertigungstiefe abbauen und sich in Hersteller von Gesamtlösungen, die Komponenten in Frameworks montieren, in Komponentenhersteller und in Subsystemhersteller aufteilen [Sche98]. Gerade die Portalindustrie kann sich nach und nach zu einer mehrstufigen Zulieferstruktur hin entwickeln - vergleichbar mit der Automobilindustrie oder mit dem Anlagen- und Maschinenbau.

System- und „Portalintegratoren“ sowie Software-Beratungshäuser werden auf der Basis vorhandener standardisierter Portaltechnologien (z. B. von IBM [Wels $\left.{ }^{+} 02\right]$, SAP [Popp02] oder anderen größeren Portalanbietern) individuelle Unternehmensportale für die betrieblichen Funktionsbereiche, Geschäftsprozesse und Querschnittsbereiche mit Hilfe funktionsspezifischer Portlets konfigurieren und für den Kunden anpassen. Sie agieren als Systemlieferanten und Integratoren an der Schnittstelle zwischen Portlet-Zulieferern, Technologielieferanten und den PortalAnwenderbetrieben.

Bei der Konfiguration von Portalen ist es möglich, Anwendungsbausteine von unterschiedlichsten Softwareanbietern miteinander zu kombinieren und diese in das geplante Unternehmensportal zu integrieren. Dies bedeutet eine Chance für kleine- 
re und mittlere Softwarehäuser (SW-KMU), ihre meist branchenspezifischen Lösungen auch in Portale bei Großunternehmen einzubinden. Bislang hatten gerade SW-KMU Schwierigkeiten, ihre Anwendungen direkt an die großen Anwender (=Endkunden) der Portale zu liefern. Eine portal-orientierte Standardisierung der Portlets wirkt dabei unterstützend auf den Vertrieb und die Wartung von Software-Modulen beim Endkunden.

Die folgende Abbildung 2 skizziert die Portal-Wertschöpfungskette einer künftig denkbaren mehrstufigen Zulieferstruktur der Softwarebranche in komplexen Portalprojekten:

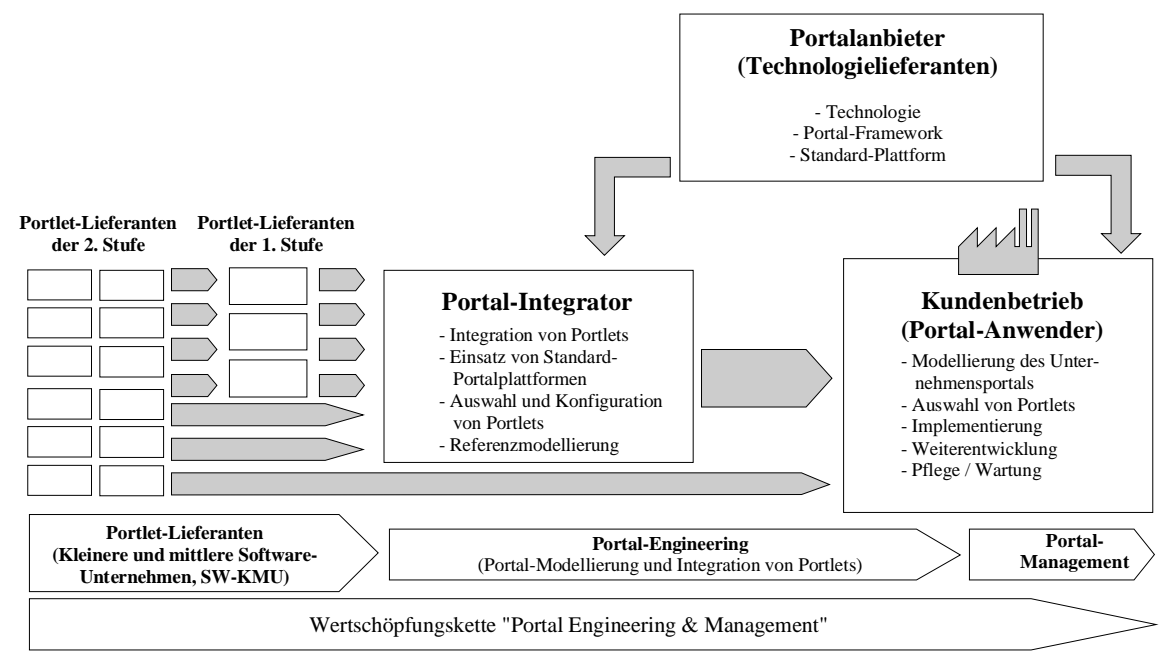

Abbildung 2: Mehrstufige Wertschöpfungskette bei der Konzeption und Implementierung komplexer Unternehmensportale

1. SW-KMU, welche in der Lieferkette weiter hinten stehen $(>=2$. Stufe), liefern ihre Module und Funktionsbausteine entweder an die erste Lieferstufe, d. h. an größere SW-Unternehmen, an den Portalintegrator oder direkt an den PortalAnwenderbetrieb. Hierzu benötigen die SW-KMU entsprechende PortletStandards in Form von „Redbooks“ oder Leitfäden, die ihnen bestimmte Verfahrensweisen und Richtlinien für die Entwicklung der Anwendungsbausteine vorgeben.

2. Die Portlet-Lieferanten der 1. Stufe integrieren Funktionsbausteine zu größeren webbasierten, branchenorientierten Anwendungsbausteinen, die entweder an die Portalintegratoren oder unmittelbar an den Endkunden geliefert werden können. Dies können Portlets für e-Commerce, das Wissensmanagement oder für die Zusammenarbeit der Mitarbeiter (Collaboration) im Betrieb sein. 
3. Portalintegratoren sind - ähnlich wie die Systemzulieferer in der Automobilindustrie - Lieferanten von kompletten Systemkomponenten. Sie übernehmen den „Einbau“ funktionsreicher Systemmodule vor Ort beim Kunden, d. h. die Konzeption und Installation von Portalen und der benötigten Portlets, und begleiten das Projektmanagement. Portalintegratoren benötigen vor allem Modellierungswerkzeuge und eine Portlet-Datenbank, in der die verfügbaren Portlets von Fremdherstellern nach bestimmten Anforderungskriterien verschiedener Kundenbetriebstypen recherchiert werden können.

4. Der jeweilige Kundenbetrieb stellt Anforderungen an das Portal, die von seiner Branche oder seinem Betriebstyp bestimmt werden. Vorgefertigte ReferenzAnwendungsarchitekturen (Portalmodelle) erleichtern es ihm, Unternehmensportalprojekte schnell, effizient und kostengünstig durchzuführen.

\section{Anforderungen an ein Portal-Engineering}

Portal-Engineering umfasst die Anwendung wissenschaftlicher Erkenntnisse und Verfahren auf die „Konstruktion“ von Portalen. Der Begriff enthält zudem die Forderung, mit ebenso gesicherten und erprobten Techniken zu operieren wie die traditionellen Ingenieursdisziplinen. Portal-Engineering umfasst als spezielle Ausprägung des Software-Engineering folgende Problembereiche: Entwicklung neuer Portale, Wartung und Weiterentwicklung bestehender Portale, Management des Entwicklungsprozesses und die Qualitätssicherung. Im Zentrum des integrierten Portal-Engineering stehen Vorgehensmodelle, Methoden, Werkzeuge und Referenz-Anwendungsarchitekturen, die ein systematisches, ingenieurmäßiges Vorgehen bei der Konzeption, Implementierung und Konfiguration von integrierten Portalen gewährleisten sollen.

Für diese Arbeit wird der Fokus auf Instrumente zur Entwicklung von Portalen auf der Basis kommerzieller Standardportalprodukte, wie z. B. von IBM, SAP oder Hyperwave gelegt. Instrumente zur Individualentwicklung werden explizit ausgegrenzt. Damit wird insbesondere die bereits vorgestellte mehrstufige Wertschöpfungskette berücksichtigt, die wesentlich auf Plattformunabhängigkeit, Standards, Wiederverwendbarkeit und Offenheit ausgerichtet ist. Der Schwerpunkt liegt auf der Ermittlung von Anforderungen an Instrumente in den frühen Phasen der Entwicklung, insbesondere in der Anlayse- und Designphase. Gerade die modellgestützte Entwicklung durch eine formalisierte Zwischenrepräsentation ist für die Untersuchung und Entwicklung komplexer Systeme hilfreich [Ambe99]. Ein weitere Forderung ergibt sich aus dem Begriff des Portal-Engineering, der auf eine möglichst enge Integration aufeinander abgestimmter Vorgehensmodelle, Methoden und Werkzeugen zielt. 
Um die verschiedenen Anforderungen an ein Portal-Engineering in eine Ordnung $\mathrm{zu}$ bringen, werden die Anforderungen weiter unterteilt. Im Vordergrund stehen die „W-Fragen“ nach dem Vorgehen (Was ist wann von wem zu tun?), den Methoden (Wie ist etwas zu tun?) und der Werkzeugunterstützung (Womit ist was zu tun?). Damit können die verschiedenen Anforderungen an Lösungs- und Entwicklungsansätze präzisiert und geordnet werden [Ambe99]; [Remu02].

\subsection{Anforderungen hinsichtlich Methodik}

Methoden regeln im Kontext der Systementwicklung, wie etwas zu tun ist. Bezogen auf die Modellierung geben Methoden an, welche Inhalte während der Durchführung der Aktivitäten wie, d. h. unter Verwendung welches Begriffssystems, zu betrachten und abzubilden sind [Ambe99]. Die Entwicklung betrieblicher Informationssysteme, wie z. B. Mitarbeiterportale, ist ohne Methodenanwendung nicht effektiv möglich.

\section{Modellierung verschiedener Sichten}

Bei der Entwicklung von Portalen sind verschiedene Sichten miteinander zu kombinieren. Es müssen z. B. Portletstrukturen, Benutzer, Informationsanforderungen und -quellen modelliert werden können. Modellierungsmethoden sowie werkzeuge für Portale sollten die Abbildung der vielfältigen Teilsichten unterstützen.

Ein nach Sichten strukturiertes Architekturkonzept kann die Modellierung von integrierten Unternehmensportalen vereinfachen. Ziel ist es, ein vollständiges Modell als Vorlage für die Implementierung des integrierten Unternehmensportals und seiner Sub-Portale zu generieren. Die Portalarchitektur kann dabei in vier verschiedene, logische Sichten zerlegt werden, welche jeweils in separaten Projektschritten modelliert werden können. So entstehen nach und nach Teilmodelle für die Benutzergruppen, die Informationsstrukturen und Informationsquellen sowie für die verwendeten Anwendungsbausteine (Portlets). Dadurch besteht die Möglichkeit, einzelne Sichten durch besondere Methoden zu beschreiben, ohne jeweils die Zusammenhänge zu den anderen Methoden einbeziehen zu müssen. Anschließend werden aber die Verbindungen zwischen den Sichten wieder aufgenommen.

Die bei einem Unternehmensportal zu beschreibenden Komponenten einschließlich ihrer Beziehungen untereinander können logisch unterteilt werden in Benutzergruppen, Informationsanforderungen (Informationsstrukturen), Informationsquellen und Anwendungsbausteine (Portlets). Für jede dieser Teilsichten kann ein eigenständiges Teilmodell entwickelt werden. Erst die Zusammenführung der Teilmodelle in einem Portal-Gesamtmodell ergibt eine integrierte Architektur der zukünftigen Wissens- und Informationslogistik im Unternehmen (vgl. Abbildung 3). 
Ein zweiter Grundgedanke neben dem Zerlegungsprinzip in verschiedene Sichten ist das Konzept unterschiedlicher Beschreibungsebenen. Portale können in unterschiedlicher Nähe zur jeweils verfügbaren Portaltechnologie beschrieben werden. So ließe sich jede Sicht über mehrere Beschreibungsebenen hinweg schrittweise der realen Portalarchitektur annähern. Im ARIS-Ansatz beispielsweise werden Methoden hinsichtlich Fachkonzept, DV-Konzept sowie Implementierung unterteilt (vgl. z. B. [Sche98]). Die Verknüpfung der Teilmodelle sollte schließlich im integrierten Portalmodell erfolgen, das die Vorlage für die Realisierung mit Hilfe einer marktgängigen Portalplattform bildet.

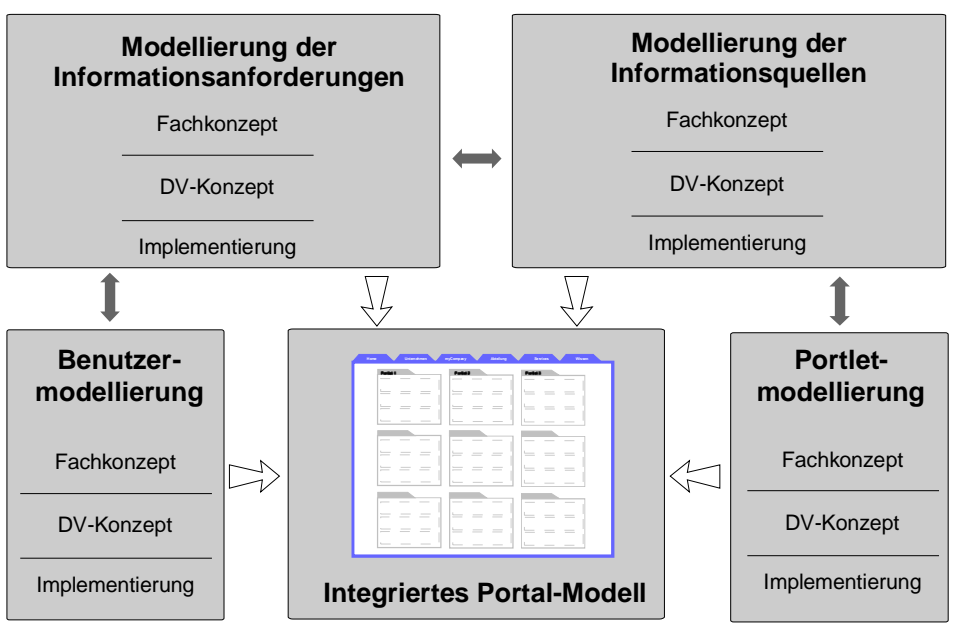

Abbildung 3: Relevante Sichten und Beschreibungsebenen beim Portal-Engineering

Die Teilmodelle werden nach und nach vervollständigt, $d$. h. dass immer wieder zu einer Sicht zurückgekehrt werden kann, wenn es beispielsweise sinnvoller erscheint, einzelne Fachgruppen noch differenzierter mit Informationen zu versorgen oder zusätzliche Portlets zur Verfügung zu stellen. Mit der Modellierung der jeweiligen Teilsichten sind folgende Tätigkeiten verknüpft:

\section{Benutzermodellierung}

In dieser Teilsicht werden die Benutzerstrukturen festgelegt (vgl. z. B. [SAP00]). Die späteren Informationsempfänger, Benutzergruppen und Anwender des Unternehmensportals wie z. B. Mitarbeiter, Werke, Abteilungen oder Fachgruppen sowie externe Benutzer wie Lieferanten, Kunden oder Investoren müssen sinnvoll gruppiert werden. Alle mit den Benutzern verbundenen Festlegungen (Rechtevergabe, Single Sign On etc.) sind Teil dieser Modellierungssicht. 


\section{Modellierung der Informationsanforderungen}

Für jede Benutzergruppe sind im Zuge der Informationsstrukturierung die Bedürfnisse und Anforderungen an die Verteilung der Daten, der Informationen und des betrieblichen Wissens zu modellieren. Hierfür werden verschiedene Themenbereiche und die vorhandenen Geschäftsprozesse des Unternehmens systematisch analysiert. Der Informationsbedarf jeder Benutzergruppe bezüglich jedes Themenbereichs (z. B. Qualitätsmanagement, e-Learning, Technologien, Produkte etc.) und jedes Geschäftsprozesses (z. B. Auftragsdurchlauf, Produktionslogistik, Reklamationen etc.) wird festgelegt und dient als Ausgangspunkt für die nachfolgende Ermittlung der Informationsquellen.

\section{Modellierung der Informationsquellen}

Die vorige Teilsicht der Informationsanforderungen und -strukturen beschreibt die Anforderungen der künftigen Portalnutzer an die „Informationslieferanten“. Für jedes Anforderungselement ist deshalb zu untersuchen, woher die Daten, die Informationen und das Wissen geliefert werden können, welche Informationsquellen also zur Verfügung stehen bzw. erst noch zu schaffen sind.

Das Teilmodell "Informationsquellen" beschreibt somit alle Parameter der datenliefernden Anwendungen, der zugrundeliegenden internen und externen Datenbanken sowie weiterer notwendiger Daten- und Informationsquellen.

Bei komplexen Informationsstrukturen kann das resultierende Teilmodell überdies in Verbindung mit der Portlet-Modellierung als Basismodell für den Einsatz und die Implementierung eines Content Management-Systems - ergänzend zur PortalSoftware - herangezogen werden.

\section{Modellierung von Anwendungsbausteinen}

Das Teilmodell „Informationsquellen“ liefert die Vorgaben für die Ermittlung der Anwendungsbausteine (Portlets), die letztendlich alle benötigten Funktionen für die Informationsversorgung der Benutzergruppen bereitstellen, so z. B. Reports, Links, Dokumente, grafische Auswertungen etc.

Während der Portlet-Modellierung sollte auf vorgefertigte Anwendungsbausteine zugegriffen werden, die beispielsweise in einer Portlet-Datenbank bereitliegen oder von herstellerspezifischen Portlet-Marktplätzen bezogen werden können. Die Portlets werden anschließend konfiguriert, d. h. das Projektteam legt detailliert die Eigenschaften und Attribute des jeweiligen Anwendungsbausteins fest.

In dieses Teilmodell fließen Kosten- und Nutzen-Betrachtungen zu jedem Portlet ein; auch Prioritäten für die Reihenfolge der Portlet-Realisierung sollten vergeben werden. 


\section{Verknüpfung der Teilmodelle zur integrierten Portalarchitektur}

In der integrierten Portalarchitektur werden die Teilsichten schließlich zusammengeführt, um ein Gesamtmodell für die Implementierung und Konfiguration des betriebsspezifischen Unternehmensportals unter Einsatz einer am Markt verfügbaren Standardsoftware zu generieren. Dabei können Portal-Referenzmodelle und vorgefertigte Portlet-Packages sowie die in Abschnitt 0 skizzierte Portlet-Datenbank die integrative Rolle spielen. In der intergrierten Portal-Architektur werden die in der Portlet-Sicht modellierten Portlets mit den modellierten Informationsstrukturen (Informationsanforderungen und -quellen) und Benutzersichten (Rollen) zu einem Gesamtmodell verknüpft. Informationsbeziehungen zwischen Portlets innerhalb von Portlet-Packages können weiter modelliert und auch Rollen mit Portlets verknüpft werden.

\subsection{Anforderungen hinsichtlich der Vorgehensweise}

Vorgehensmodelle legen fest, welche Aktivitäten durchzuführen sind, welche Ergebnisse dabei zu produzieren sind und welche Inhalte diese Ergebnisse haben müssen. Ein wesentlicher Bestandteil ingenierurmäßigen Vorgehens, wie dies ein ausgereiftes Portal-Engineering erfordert, ist eine wiederholbare, nachvollziehbare und dokumentierte Vorgehensweise, die reproduzierbare und qualitätsgesicherte Entwicklungen sicherstellt [Ambe99].

An ein integriertes Unternehmensportal werden gewisse Mindestanforderungen gestellt, die sich mit dem vorherrschenden Vorgehen bei Portalprojekten (,Trial and Error") nicht erfüllen lassen. Die Erweiterbarkeit durch neue Portlets und Webservices, die Integrationsfähigkeit von Fremdlösungen und fremdbezogenen Portlets sowie die flexible Anpassung an die Kundenbedürfnisse sollte sichergestellt werden.

Es existieren bereits einige Vorgehensmodelle zur Entwicklung und Einführung von Portalen (vgl. z. B. [Scal02], [Gurz02], [IMG02]). Ausgangspunkt ist häufig die Definition einer Portalstrategie, die als Ziel die Entwicklung von Portalideen, Alleinstellungsmerkmalen, Wettbewerbsfaktoren und die Verknüpfung zur Unternehmensstrategie hat. Ein nächster Schritt ist die Analyse der bestehenden Prozesslandschaft, der Organisation, der beteiligten Anwendungssysteme und weiterer Ressourcen. Dazu gehört auch eine Wirtschaftlichkeitsanalyse. In der anschließenden Konzeptionsphase wird die IT-Architektur entwickelt, die PortalSoftware und der Technologieanbieter ausgewählt sowie das Pflichten- und Lastenheft erstellt. Die Realisierung erfolgt häufig durch Prototyping, um bei den Mitarbeitern "Quick Wins", d. h. kleine, aber rasch erkennbare Erfolge, zu realisieren. Schließlich wird ein Einführungskonzept entwickelt und die PortalSoftware organisatorisch und technologisch implementiert. Die Einführungsphase wird durch ein Change Management-Programm begleitet, während das PortalControlling dafür sorgt, dass das Anwendungssystem auf der Basis eines regelmä- 
Bigen Feedbacks durch die Benutzer kontinuierlich verbessert und weiterentwickelt werden kann.

Aufbauend auf den Anforderungen hinsichtlich der Methodik sollte sich ein Vorgehensmodell für ein Portal-Engineering an ein Modell anlehnen, das es dem Projektteam ermöglicht, ähnlich wie bei anderen SW-Engineering-Projekten iterativ voranzuschreiten und sich allmählich dem endgültigen Portalmodell anzunähern (vgl. Abbildung 4). Die Architektur integrierter Unternehmensportale mit ihren verschiedenen Sichten spielt hier die integrative Rolle.

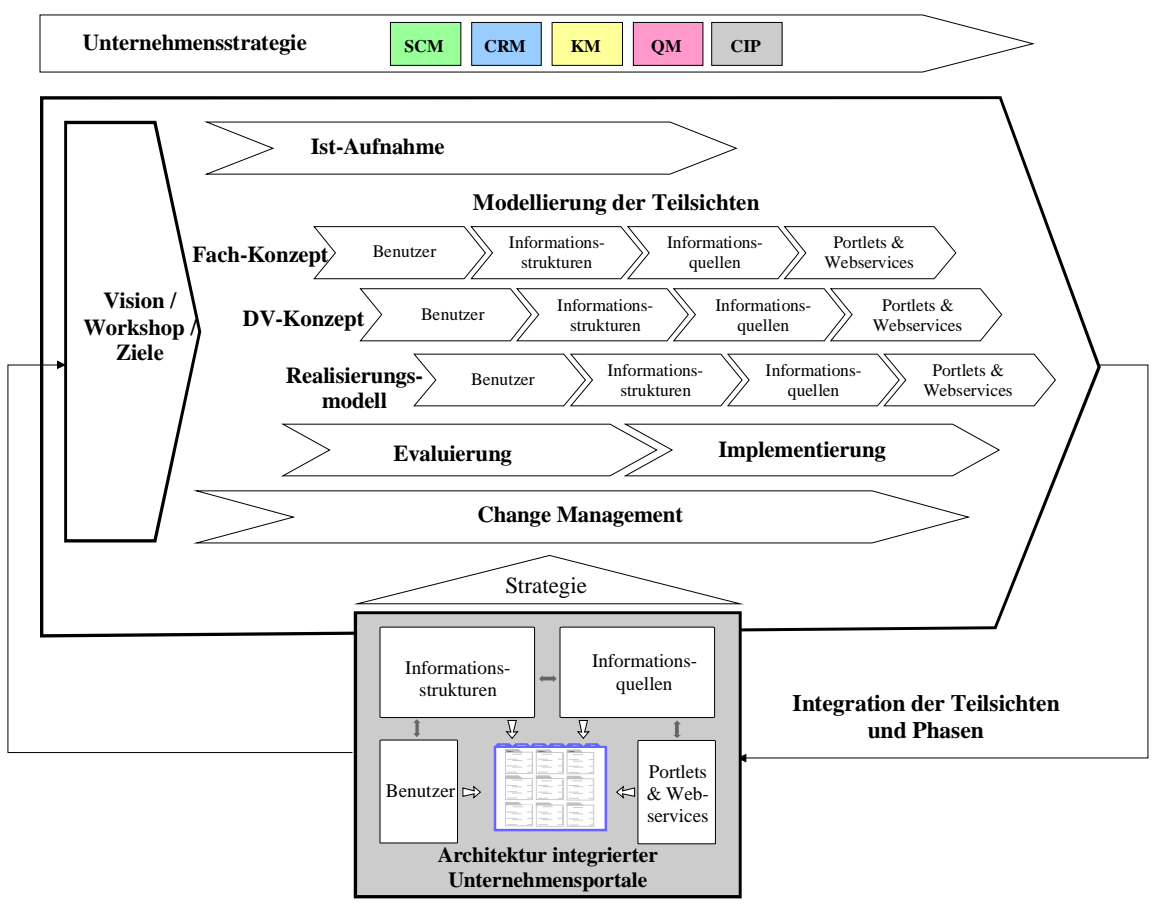

Abbildung 4: Vorgehensmodell bei der Konzeption und Implementierung von Unternehmensportalen

Im ersten Schritt wird die Unternehmensstrategie im Bereich der Unternehmensportale festgelegt und definiert. Meist greift man einen oder mehrere Schwerpunkte heraus, die dem Anwenderbetrieb die größte Rentabilität oder den größten Effizienzgewinn versprechen, wie z. B. B2B-Portale, Mitarbeiter- oder Kundenportale.

In der Ist-Analyse werden die Ist-Prozesse und -Funktionen ermittelt sowie eine erste Anforderungsanalyse erstellt. Über die gesamte Projektlaufzeit werden die Ist-Prozesse und deren Fortschritte hinsichtlich Prozess-Verkürzung und -Verbesserung dokumentiert. Auf der Grundlage der oben beschriebenen Modellierungssichten generiert das jeweils verantwortliche Portalteam in mehreren Itera- 
tionen ein Fach- und DV-Konzept sowie das Realisierungsmodell für das später zu implementierende, integrierte Portal.

Begleitend zur Modellierung beginnt der Vergleich von Portal-Standardsoftware und -Technologien, die je nach Komplexität und Kundenanforderung in Frage kommen (Evaluierungsphase).

\subsection{Anforderungen hinsichtlich Werkzeugunterstützung}

Software-Werkzeuge stellen Funktionen bereit, die bei den einzelnen Aktivitäten des Entwicklungsprozesses verwendet werden können. Die Entwicklung betrieblicher Informationssysteme in praxisrelevanten Grössenordnungen ist ohne eine solche Unterstützung nicht effizient möglich.

Software-gestützte Werkzeuge für ein Portal-Engineering sollten insbesondere folgende Komponenten umfassen:

1. Portal-Referenzmodelle und vorgefertigte Portlet-Packages für bestimmte Branchen oder Benutzergruppen,

2. "Portlet-Checklisten" für bestimmte betriebliche Benutzergruppen,

3. die Erstellung und Pflege einer Portlet-Datenbank mit umfangreichen Deskriptoren zur Erfassung verschiedenster Anwendungsbausteine sowie

4. vorkonfigurierte Portale für Unternehmen bestimmter Branchen oder Betriebstypen.

Gerade kleinen und mittelständischen Softwareunternehmen mangelt es an „Portal-Richtlinien“, um standardisierte, integrationsfähige Portlets - u. a. auch aus ihren bestehenden Anwendungslösungen - generieren zu können. Eine ständig aktualisierte, internetbasierte Portlet-Datenbank, die den Portalherstellern, den Anwenderbetrieben und den Softwareunternehmen sowie den Portalintegratoren zur Verfügung stünde, könnte den Entwurfs- und Modellierungsprozess in Portalprojekten erheblich erleichtern.

Die Instrumentarien werden nachfolgend im Überblick beschrieben:

\section{Branchen-Portalmodelle}

Referenzmodelle sind vorgefertigte Templates, Frameworks oder „PortalSchablonen" für bestimmte Anwenderzielgruppen, wie Branchen, Betriebstypen oder innerbetriebliche Benutzergruppen mit zugewiesenen Rollen. Ein Instrument zum Portal-Engineering sollte daher allgemeingültige Portal-Referenzmodellen für Industrie-, Handels- und Dienstleistungsbetriebe und für ausgewählte Branchen anbieten. Auf der Basis solcher Modelle spart der Anwenderbetrieb bei seinem Portalvorhaben Zeit durch Nutzung von wiederverwendbaren Vorlagen ("PortalSchablonen") insbesondere in der Konzeptionsphase. 
Portalintegratoren können bei der Modellierung und Implementierung von Unternehmensportalen auf vorgefertigte Portlets oder komplette Pakete mit fach- oder branchenspezifischen Anwendungsbausteinen zurückgreifen, um diese in das spätere Unternehmensportal einzufügen. $\mathrm{Zu}$ den Referenzmodellen zählen daher auch vorgefertigte Portlet-Sammlungen ("Portal Business Packages"), die den Einstieg in die Portalkonzeption und -modellierung erleichtern und das Projekt schneller voranbringen.

\section{Portlet-Checklisten}

Von Nutzen für die Modellierung der Teilsichten "Benutzergruppen" und "Informationsstrukturen" sind Portlet-Checklisten für Fach- und Führungskräfte, die in verschiedene betriebliche Funktionsbereiche und Geschäftsprozesse eingebunden sind. Die Portlet-Checklisten enthalten Vorschläge für Anwendungsbausteine und Informationselemente, die die administrativen und dispositiven Tätigkeiten der jeweiligen Benutzergruppe und ihre Controlling- und Planungsaufgaben unterstützen können. Mit Hilfe der Checklisten lässt sich prüfen, ob die Informationsversorgung über das Portal dem jeweiligen State-of-the-Art entspricht. Fehlende Informationsbausteine können auf diese Weise schneller ermittelt werden.

\section{Portlet-Datenbank}

Die Portlet-Datenbank ist eine strukturierte Sammlung von bestehenden Portlets und deren Deskriptoren mit der Möglichkeit, diese Portlets zielgerichtet zu suchen und nach verschiedenen Kriterien flexibel zu sortieren. Portlets sind Informationsund Softwarebausteine wie z. B. Links, Applikationen, Reports, Dokumente und Formulare, Templates und Best Practice-Module, die in Unternehmensportale integriert werden können. Die Portlets brauchen nicht in jedem Fall als vollständig "ausprogrammierte" Softwarebausteine vorliegen: möglich sind beispielsweise auch Links zum Download der Bausteine, Beschreibungen von Informationsbausteinen, Templates (=Software-Schablonen) oder Software-Module, die parametrisierbar sind.

Portlets werden mit Hilfe von Deskriptoren in der Datenbank ,verschlagwortet“. Hierzu gehören beispielsweise folgende Deskriptoren:

- Kategorie (Link, Applikation, Dokument, Report, News, Groupware / Collaboration, Kommunikation, Workflow, Internet-Marktplatz, ...)

- Realisierungsstand (Software-Template, parametrisierbares Tool, verfügbare Software-Bausteine von Fremdanbietern, ...)

- Inhalt / Funktionsweise des Portlets

- Schnittstellen zu Fremdsystemen wie z. B. ERP-Systeme, Business Intelligence-Anwendungen oder MIS 
- Einsatz- und Funktionsbereich im Unternehmen (Beschaffung, Personal, Vertrieb/Marketing, Produktion, Rechnungswesen, ...)

- Festlegung der erforderlichen Schnittstellen und Datenquellen zur Aktualisierung des jeweiligen Portlets

- Kosten zum Kauf oder Aufwand zur Realisierung der Komponente

- Portlet-Bezugsquelle

- erzielbare Nutzeffekte beim Einsatz des Portlets

\section{PreBuilt Portals}

PreBuilt Portals verbinden die Vorteile von Standardsoftware mit den Vorzügen von individuell entwickelten, branchen- oder betriebstypspezifischen Funktionsund Anwendungsbausteinen. In einem PreBuilt Portal sind vorgefertigte Applikationen nach dem ,,best-of-breed“-Ansatz integriert wie beispielsweise Dokumenten-Management-Systeme, Befragungstools oder ausgefeilte Recherchetools (Suchagenten) sowie Anwendungen für das Projektmanagement.

\section{Ausgewählte Ansätze zum Portal-Engineering}

In diesem Kapitel werden ausgewählte Ansätze zum Portal-Engineering in Bezug auf die in Kapitel 3 aufgestellten Anforderungen diskutiert. Die Auswahl berücksichtigt ausschließlich Lösungsansätze zur Modellierung kommerzieller, standardisierter Portalsoftware, wie sie etwa von SAP, IBM oder Hyperwave angeboten wird. Zudem sollten sie möglichst alle genannten Aspekte des Portal-Engineering unterstützen und nicht nur einen Aspekt, z. B. die Konfiguration oder Implementierung.

\subsection{Lösungsansatz von SAP: Business Packages}

Die SAP AG ermöglicht es, auf der Basis ihrer Portaltechnologie sogenannte " $\mathrm{Bu}$ siness Packages" für ausgewählte Branchen, Benutzergruppen, betriebliche Funktionsbereiche oder innerbetriebliche Rollen (z. B. Manager oder Fachkraft) als vorgefertigte "Portlet-Bundles" einzusetzen. Bei den SAP Business Packages handelt es sich somit um inhaltlich aufeinander abgestimmte Sammlungen von Portlets zur Unterstützung der Informations- und Wissensverarbeitung für die jeweilige Zielgruppe. Die Modellierung und Implementierung der Portlets im Rahmen von sogenannten Worksets (inhaltlich zusammenhängende Portlets) wird auf diese Weise stark vereinfacht. Allerdings werden von SAP keine Hilfsmittel angeboten, die vorhergehenden Phasen des Portal-Engineering aus den verschiedenen Teilsichten der Portalarchitektur in zufriedenstellender Form zu unterstützen. Die SAP 
Business Packages sind daher als eine Mischform zwischen Implementierungsmodellen der Teilsicht "Anwendungsbausteine" (vormodellierte PortletImplementierungen auf der Beschreibungsebene "Realisierung") und branchenbzw. benutzergruppen-spezifischen PreBuilt Portals (vorgefertigte "Worksets" für bestimmte Rollen und Benutzergruppen) anzusehen.

\subsection{Lösungsansatz von IBM: Portlet Marketplace}

IBM stellt eine umfangreiche Datenbank vorgefertigter, standardisierter und implementierungsfähiger Anwendungsbausteine (Portlets) zur Verfügung. Über einen internetbasierten Portlet-Marktplatz können die Softwaremodule bezogen werden; Zielgruppen sind Portalintegratoren und Anwenderbetriebe. In einer PortletDatenbank kann mithilfe zahlreicher Deskriptoren in Tausenden von Anwendungsbausteinen recherchiert werden. Zu den Deskriptoren zählen beispielsweise die Einsetzbarkeit eines Portlets für bestimmte Branchen, die genaue Definition seines Anwendungsbereichs im Betrieb (Benutzergruppen, Funktionsbereiche und Geschäftsprozesse) oder die Zuordnung zu bestimmten Aufgabengebieten (Wissensmanagement, Enterprise Resource Planning, Supply Chain Management). Die Schnittstellen der Portlets sind standardisiert, so dass die software-technische Integration in Portal-Frameworks erleichtert wird.

IBM liefert mit der Portlet-Datenbank einerseits ein wesentliches Instrument zur schnellen Konfiguration von Unternehmensportalen. Die Teilsicht "Anwendungsbausteine" des Architekturmodells für integrierte Unternehmensportale kann damit auf der Ebene "Realisierung / Implementierung" effizient unterstützt werden. Andererseits wird aber die Modellierungsphase vollständig ausgeblendet; erst in der Phase der Auswahl bestehender Portlets wird das Portal-Engineering durch das Instrumentarium der IBM ausreichend unterstützt.

\subsection{Lösungsansatz von IDS Scheer: ARIS for Hyperwave}

Mit ARIS for Hyperwave ${ }^{\circledR}$ bietet der Marktführer von Prozessmodellierungstools, die IDS Scheer AG, eine Werkzeugunterstützung bei der Konfiguration von Hyperwave Information Portals an. Mit Hilfe dieses Werkzeugs können Prozessmodelle und Rollenmodelle aus ARIS in das Hyperwave Information Portal als Navigationsstruktur bzw. als Rollen und Rechte importiert werden. Zusätzlich können in Hyperwave Prozessaktivitäten zu Wissenskategorien zugeordnet werden [Sche01].

Der Ansatz bietet ausserdem ein Rollen- und Rechtekonzept an, mit dessen Hilfe die Portalstruktur vorkonfiguriert wird. Der Ansatz soll die Modellierung und Strukturierung sämtlicher Objekte eines Portals (Anwender, Rollen, Rechte, Abläufe und Inhalte) unterstützen. Der Teilnehmer kann dann das auf seine Rolle bereits zugeschnittene Portal weiter personalisieren. In ARIS modellierte Rollen 
können zukünftig durch das Werkzeug ARIS for Hyperwave ${ }^{\circledR}$ direkt in die Hyperwave Rollen- und Rechte-Datenbank übernommen werden.

Zusätzlich wird eine Methodik zur Portal-Entwicklung angeboten. Die erste Phase dient u. a. der Erfassung der wichtigsten Prozesse, der Systemunterstützung, der verfügbaren Inhalte sowie der Verbesserungspotenziale. In der zweiten Phase wird ein Sollkonzept erstellt, das die wesentlichen Aufgaben und Inhalte im Rahmen eines moderierten Workshops modelliert und in Hyperwave implementiert. In der Pilotierungs- und Umsetzungsphase werden die Inhalte in Form von Portlets weiter strukturiert und in der Oberfläche angeordnet sowie das Portal schließlich beim Anwender eingeführt.

Referenzmodelle für einzelne Branchen (Versicherung, Einzelhandel, Reiseberatung) werden nur rudimentär in Form von Beispielszenarien angeboten. Innerhalb von ARIS können die vom Portal zu unterstützenden Prozesse, Organisationsstrukturen, Rollen, Funktionen Anwendungssysteme, Daten und Informationsstrukturen modelliert werden. Die automatische, werkzeuggestützte Übernahme aller Modellierungsergebnisse in einen Portal-Prototyp ist aber nicht möglich. Es fehlen außerdem Methoden zur Modellierung von Portlets und eine integrative Modellierung des Gesamtportals aus den jeweiligen Einzelsichten.

\subsection{Lösungsansatz von Visible Systems Corp.: Business Templates}

Finkelstein und Perkins (Visible Systems Corp.) beschreiben ein detailliertes Vorgehensmodell für das Portal-Engineering, insbesondere die Phasen zur Konzeption und Modellierung eines Enterprise-Portals [Fink02]. Der inhaltliche Fokus liegt auf Portalen mit Anwendungsbausteinen für Data Warehouse- und Business Intelligence-Auswertungen. Visible Systems Corp. bietet für das Portal-Engineering in diesem Bereich (Reporting) vorgefertigte "Business Templates" an, die die Entwicklung von Portal-Modellen wesentlich beschleunigen sollen: "Business Templates are high-level data models of nearly 50 business objects an encompassing over 400 entities with 1.000 attributes. Business Templates models also contain hundreds of common enterprise functions linked to the business data objects that typically support them" [Fink02, S. 8].

Visible Systems unterteilt die Modellierung in folgende vier Sichten, die sich teilweise mit den oben dargestellten Teilsichten der "Architektur integrierter Unternehmensportale" überschneiden: Business Architecture (Strategiefindung, Abgrenzung der Funktions- und Informationsbereiche sowie der Benutzergruppen), Business Model (Definition der Informationsstrukturen und -anforderungen), Data Architecture (Zuordnung von Informations- und Datenquellen zu den Benutzergruppen) und Application \& Technology Architecture (Anwendungen und Softwarebausteine für das zuvor erarbeitete "information system design") [Fink02, S. $11 \mathrm{f}$.$] .$ 
Methodiken und Werkzeuge für die einzelnen Phasen werden nicht eingehender beschrieben; jedoch findet sich der Hinweis, dass das Software-Unternehmen $V i$ sible Systems Corp. entsprechend Tools zur Unterstützung der einzelnen Phasen anbietet. Vorgefertigte Modelle werden in Form der Business Templates bereitgehalten; sie beschränken sich funktionell jedoch stark auf Anwendungen im Bereich Knowledge Management.

\subsection{Zusammenfassende Betrachtung}

Bei der näheren Betrachtung bestehender Ansätze fällt folgendes auf: Alle hier diskutierten Ansätze sind produktbezogen und lassen sich nicht oder nur schwer für andere Portalsoftware verwenden. Standards für Portlets und Portalsoftware könnten zur Lösung dieses Problems wesentlich beitragen. Zudem berücksichtigen die Ansätze häufig nur eine Stufe in der Portal-Wertschöpfungskette, wie z. B. Portal-Integratoren oder Portal-Anwender. Die Identifikation und Integration von Portlets fremder Hersteller ist aufgrund fehlender Transparenz und Suchmöglichkeiten häufig nur schwer möglich. Modellbasierte Ansätze bilden eher die Ausnahme. Auch ARIS for Hyperwave bietet keine detaillierten (Branchen)Referenzmodelle zur modellbasierten Konfiguration des Hyperwave Information Portals an. Zudem werden nicht alle Sichten berücksichtigt (z. B. die Sicht auf Portlets); auch eine integrative Portalsicht wird nicht angeboten. Nur wenige Lösungsansätze bieten ein abgestimmtes Vorgehen zum Portal-Engineering an. Die aufgezeigten Lücken können durch die Entwicklung eines integrierten Instrumentariums zum Portal-Engineering geschlossen werden. Die dazu notwendigen Forschungs- und Entwicklungsarbeiten werden im folgenden Kapitel 5 skizziert.

\section{Diskussion und Ausblick}

Unternehmensportale bieten auf der einen Seite hohe Nutzenpotenziale, wie z. B. einen einheitlichen Zugriff auf unternehmensinternes und -externes Wissen, vielfältige Bearbeitungs- und Interaktionsmöglichkeiten, Möglichkeiten zur individuellen Gestaltung (Personalisierung) sowie eine Anpassung auf den jeweiligen Handlungskontext. Auf der anderen Seite sind Portale aufgrund ihrer Komplexität, hervorgerufen durch die Vielzahl unterschiedlicher Portlets, die Vielzahl unterschiedlicher Portalkategorien, Anwendungsbereiche und Sichten, häufig sehr schwierig zu entwickeln und anzupassen. Ein weiteres Problem entsteht durch die Berücksichtigung der verschiedenen Akteure im Entwicklungsprozess im Rahmen einer übergeordneten Wertschöpfungskette, welche sich von der Entwicklung einzelner Portlets (Anwendungsbausteine) über deren Integration in ein Portalframework bis hin zur Einführung und Inbetriebnahme des fertigen Portals im Kundenbetrieb erstreckt. Hersteller bieten einerseits standardisierte Portal-Frameworks 
und Portlets an, andererseits müssen diese erst strukturell und inhaltlich in einem zweiten Schritt an die spezifischen Unternehmensanforderungen angepasst werden. Die Portal-Implementierung muss daher Methoden der Entwicklung von Individualsoftware mit Methoden zur Einführung von Standardsoftware verknüpfen.

Neben Eigenentwicklungen werden verstärkt kommerzielle Systeme angeboten, die eine einfache Zusammenstellung und Konfiguration der $\mathrm{zu}$ integrierenden Anwendungsbausteine (Portlets) versprechen. Hier ist nachzuprüfen, inwieweit Modellierungs- und Entwicklungsansätze die wesentlichen Problemfelder bei der Portalkonzeption und -implementierung bereits berücksichtigen und welche weiteren Ansätze notwendig sind, um Schwachstellen bei der Entwicklung zu schliessen. Dazu wurden die wesentlichen Anforderungen gesammelt und in die Klassen Methoden-, Vorgehens-, und Werkzeugunterstützung eingeordnet. Instrumente zum Portal-Engineering müssen zum einen den hohen Komplexitätsgrad von Portalen, zum anderen die angestrebte Portal-Wertschöpfungskette berücksichtigen.

Der Modellierungs- und Engineering-Prozess sollte entlang der PortalWertschöpfungskette softwaregestützt durchgeführt werden können. Die Portlets, welche später als Anwendungsbausteine in das Unternehmensportal integriert werden, sollten über eine umfassende Datenbank recherchiert und anschließend parametrisiert werden können. SW-Hersteller könnten somit ihre bisherigen Lösungen an Portalstandards anpassen und über diese Datenbank anbieten. Systemintegratoren, die Portalprojekte durchführen, würden die Portlets dieser Anbieter in Unternehmensportale einbinden können. In die angestrebte PortalWertschöpfungskette sollen kleinere und mittlere Softwareunternehmen (SWKMU), System- bzw. Portalintegratoren (SW-Häuser oder Beratungshäuser mit DV-Schwerpunkt), Technologielieferanten (Hersteller von Standardportalen wie z. B. SAP) und Endanwender (mittelständische und große Unternehmen) integriert werden. Zudem sollte ein Instrumentarium zum Portal-Engineering branchenspezifische (z. B. Industrie, Handel, Öffentlicher Betrieb) und betriebstypische Anforderungen (z. B. Einzel-, Serien- oder Prozessfertiger) berücksichtigen und die Vorteile von Standardportalen mit der Integration individuell erstellter Portlets verbinden.

Die kurze Evaluation bereits existierender Entwicklungsansätze zum PortalEngineering zeigte, dass gerade bei der modellbasierten Entwicklung Lücken bestehen. Momentan herrscht noch ein Mangel an geeigneten Modellierungsmethoden und vorgefertigten Referenzmodellen. Zur Unterstützung der wesentlichen Aufgaben im Portal-Engineering müssen daher folgende Forschungs- und Entwicklungsziele verfolgt werden:

- Konzeption und Modellierung einer „Portal-Wertschöpfungskette“ und Definition der Prozesse innerhalb dieser Wertschöpfungskette für SW-KMU (Zulieferbetriebe), Portalintegratoren, Technologielieferanten und Anwenderbetriebe. 
- Konzeption eines Architekturmodells und der zugehörigen Modellierungsmethoden für komplexe Unternehmensportale.

- Entwicklung von softwaregestützten Modellierungswerkzeugen für ein effizientes Portal-Engineering.

- Erarbeiten branchenspezifischer und betriebstypischer ReferenzAnwendungsarchitekturen für die unterschiedlichen Portalkategorien.

- Entwicklung und Aufbau einer webbasierten Portlet-Datenbank zur Systematisierung, Kategorisierung und Deskription verfügbarer Portlets der Softwareindustrie - geordnet nach Branchen, Betriebstypen und Anwendungsfeldern mit einfachen Suchmechanismen für Portlets.

Der Nutzen eines ingenieurmäßigen Vorgehens bei der Implementierung von Portalen entsteht zunächst beim Anwenderbetrieb: Es ist zu erwarten, dass die Anwendung des Instrumentariums zum Portal-Engineering $\mathrm{zu}$ kostengünstigen, schnell durchführbaren, skalierbaren und adaptierbaren Portallösungen führt. Daher sind die Ergebnisse auch für KMU geeignet, die i.d.R. mit geringen Budgets auskommen müssen, jedoch ab einer gewissen Größe vor ähnlichen Integrationsproblemen wie Großunternehmen stehen. Langfristig wird eine höhere Flexibilität der Unternehmensportale erreicht, die es den Kunden ermöglicht, die Portale kontinuierlich weiterzuentwickeln. Überdies entsteht ein Nutzen für die Teilnehmer in der gesamten Portal-Wertschöpfungskette. Auf der Basis vorgefertigter Modelle und mehrfach einsetzbarer Portlets kann die Entwicklungszeit reduziert, die Qualität und der Integrationsgrad der Portale erhöht, der Überblick über vorhandene Portlets und die Suchmöglichkeiten nach Portlets verbessert sowie die Vorteile der standardisierten Portalsoftware mit den Vorteilen von vorgefertigten, branchenund betriebstyp-differenzierten Portlets verbunden werden.

\section{Literatur}

[Ambe99] Amberg, M.: Prozeßorientierte betriebliche Informationssysteme, Methoden, Vorgehen und Werkzeuge zu ihrer effizienten Entwicklung, Berlin 1999.

[Bach00] Bach, V.: Business Knowledge Management: Wertschöpfung durch Wissensportale, in: Bach, V.; Österle, H.; Vogler, P. (Hrsg.): Business Knowledge Management in der Praxis, Prozessorientierte Lösungen zwischen Knowledge Portal und Kompetenzmanagement, Berlin 2000, 51-119.

[BaSe00] Bakker, de C.P.; Uwe G. Seebacher, U.G.: Konzeptionelle Entwicklung von Internet-Portalen, in Information Management \& Consulting, Nr. 2, 2000.

[Baue01] Bauer, H.: Unternehemensportale. Geschäftsmodelle, Design, Technologien. Bonn 2001. 
[Delp99] Delphi Group: Enterprise Portals Shape Emerging Business Desktop. Viewpoint, Boston (MA); 26.1.1999, http://www.delphigroup.com/pubs/whitepapers-/ KM-VIEWPOINT-PORTAL-1999-01.pdf (letzter Zugriff: 8.2.01).

[Fink99] Finkelstein, C.: The Emergence and Potential of Enterprise Information Portals (EIPs), Information Engineering Services Pty Ltd (IES), 1999, URL: http://www.tdan.com/ i010fe02.htm (letzter Zugriff: 30.01.2001).

[Fink02] Finkelstein, C.; Perkins, A.: Enterprise Portal Engineering - A Visible Solution, www.visible.com (letzter Zugriff: 30.11.02),Visible Systems Corporation, Alexandria, Virginia 2002.

[Fire99] Firestone, J. M.: Defining the Enterprise Information Portal, White Paper No. 13, Executive Information Systems, Inc. 1999, URL: http://www.dkms.com/-EIPDEF.html (letzter Zugriff: 30.01.2001).

[Gurz02] Gurzki, T.: Vom Prozess zum Portal. Ein Vorgehensmodell für die Konzeption von Portalen, Präsentation auf dem Informationstag „Business- und Enterprise Portale erfolgreich gestalten“, 16. Mai 2002, http://www.portale.iao.fhg.de (letzter Zugriff: 13.11.02).

[Hazr00] Hazra, T.K.: Enterprise Modeling, Methodologies and Processes, OMG UML Workshop, Nov. 6-9.2000, Palm Springs, http://wwwomg.org/news/meetings/workshops/proceedings.htm (letzter Zugriff: 2.12.02).

[Hind02] Hinderer, H.: Portale in der E-Business-Strategie: Konzepte für den erfolgreichen Einsatz, Präsentation auf dem Informationstag „Business- und Enterprise Portale erfolgreich gestalten“, 16. Mai 2002, http://www.portale.iao.fhg.de (letzter Zugriff: 13.11.02).

[IMG02] IMG: Enterprise Portals - Vorgehen, White Paper, http://www.promet-web.com (letzter Zugriff: 10.11.02).

[Jans00] Jansen, C.: Prozeßunterstützung durch Wissensplattformen für Business Engineers, Diss. Universität St. Gallen, Bamberg 2000.

[Kim ${ }^{+}$02] Kim, Y.J.; Chaudhury, A.; Rao, H.R.: A Knowledge Management Perspective to Evaluation of Enterprise Information Portals, in: Knowledge and Process Management, Vol.9, No.2, 57-71, 2002.

[Marg02] Margulius, D.L.: Plug-and-play portlets, white paper, in: InfoWorld, April 2002, http://www.infoworld.com/articles/fe/xml/02/04/29/020429feportaltci.xml (letzter Zugriff: 6.12.02).

[Pfif01] Pfifer, G.: 2H01 Portal Products Magic Quadrant. URL: http://www.gartner.com/ reprints/sap/99677.html (letzter Zugriff: 6.12.01).

[Piro02] Pironet NDH AG. (HRSG.): Pironet, URL: http://www.pironet-ndh.com/servlet/ $\mathrm{PB} / \mathrm{menu} / 1002021$ (letzter Zugriff: 30.1.02).

[Plan02] Planet Internet Commerce: Regionale Virtuelle Marktplätze. URL: http://www.planet-ic.de/profil/pdf/IuK_RegMKP.pdf (letzter Zugriff: 17.4.02).

[Popp02] Popp, K.: Nutzbarmachung von Portaltechnologie: mySAP Enterprise Portals, in HMD, Praxis der Wirtschaftsinformatik, Heft 225, 2002. 
[Remu02] Remus, U. (2002), Prozessorientiertes Wissensmanagement. Konzepte und Modellierung, Regensburg 2002, http://www.bibliothek.uni-regensburg.de/ opus/volltexte/2002/80/ (letzter Zugriff: 5.2.03).

[SAP00] mySAP.com: SAP Rollen des Business Content im Business Information Warehouse, Release 2.0 A, 2000.

[Scal02] Scala Management Consulting GmbH: Portal Engineering, White Paper, http://www.scala-management-consulting.de (letzter Zugriff: 13.11.02).

[Sche98] Scheer, A.-W.: ARIS - Vom Geschäftsprozess zum Anwendungssystem, 3. Aufl., Berlin 1998.

[Sche01] Scheube, S.: ARIS for Hyperwave, Präsentationsunterlagen, IDS Prof. Scheer AG, Saarbrücken, 19.1.2001, URL: http://www.ids-scheer.de (letzter Zugriff 9.1.02).

[ScWi02] Schelp, J.; Winter, J.: Enterprise Portals und Enterprise Application Integration, Begriffsbestimmung und Integrationskonzeptionen, in HMD, Praxis der Wirtschaftsinformatik, Heft 225, 2002.

[ShTy98] Shilakes, C. C.; Tylman, J.: Enterprise Information Portals. Merrill Lynch Inc., New York 1998.

[Wels $\left.{ }^{+} 02\right]$ Welsch, M.; Dammers, R.; Bauer, W.: IBM WebSphere Portal als Basis für Unternehmensportale, in HMD, Praxis der Wirtschaftsinformatik, Heft 225, 2002. 